\title{
S3. Fundamental Ploblem in Rehabilitation After Cerebrospinal Lesions
}

-Electromyographic Evaluation of Spasticity-

\author{
Shohei Oishi, Yasuro Ozawa, Akira Iwatsu and Taira Kawata \\ Dept. of Orthopedics, Osaka Labor-Disease and Welfare Hospital \\ Masuhisa OKa \\ Dept. of Surgery, Osaka Labor-Disease and Welfare Hospital
}

Shohei MaEhara

Dept. of Orthopedics, Osaka Prefectural Hospital

Spasticity is one of main factors which hinder the performance of rehabilitation for patients with cerebrospinal lesions. Exact evaluation of spasticity should be the first step in the handling of this pathological condition.

On the trial of electromyographic evaluation of spasticity, thresholds of spinal reflex responses evoked by electric stimulation to peripheral nerves and appearances of their patterns were investigated.

Surveyed value of the threshold by percutaneous electric stimulation is varied to much extent with difference of skin-inpedance in individual. Therefore threshold ratio of reflex response to motor response was taken as indicator of excitability in the spinal reflex instead of the threshold itself. On the other hand patterns of the reflex responses were devided into four grades with types of their appearances.

Observation on patients with spinal lesions showed that these two procedures with evoked electromyography are worthy of clinical use in evaluation of spasticity.

Further investigation with these methods is proceeding to the same problem on cerebral lesions.

\section{S4. An Introduction of Philadelphia Rehabilitation Center \\ Shinken Kuramoto and Sigeyuki TAKaKI \\ Dept. of Surgery, Kurume University School of Medicine}

At the Rehabilitation Center in Philadelphia, there are the special faculties about general nursing, motility, hand function, walk, speak, sense, vision, respiration, profession, and daily training. In each field, many efforts have been made, 
under the medical doctor's directions, in order to recover the functions of patients suffering from the central nervous injuries.

The characteristics of this rehabilitation center are the fundamental researches concerning dominance of one-side hemicerebrum and performance of so-called reflex therapies in which the ideas of phylogenesis and ontogenesis are involved in rehabilitation.

It would be seen that tonic neck reflex is specially important among the reflex therapies; all of the patients suffering from central nervous injuries are daily treated with passive movements as homolateral or cross pattern (movement types of neck, hand, and foot).

I would like to introduce briefly the performance of these therapies mentioned above by using the slides.

\title{
S5. Medical Rehabilitation in Neurosurgical Practice
}

\author{
Ichiro HATTORI \\ Kyushu Rosai Hospital \\ Norihiko NAKaHARA \\ Shin Beppu Hospital \\ Katsutoshi Kitamura and Kenichi Nishimura \\ Ist Dept. of Surgery, Kyushu University
}

Medical rehabilitation was discussed dealing with sixteen cases among the patients who were treated at Kyushu Rosai Hospital or Shin Beppu Hospital after neurosurgical treatment had been done at the First Department of Surgery, Kyushu University Hospital.

As the first step of medical rehabilitation, the evaluation including muscle testing, A.D.L. testing, functional testing, range of motion, moral, social background, neurological findings, laboratory data and operative findings should be done systematically. At the end of medical rehabilitation when the recovery is supposed to be up on the plateau, prevocational evaluation, i.e., work test and physical appraisal should be done to pick out the patients who would need vocational rehabilitation.

The conclusions are briefly summarized as follows:

1. Seven patients ( $43.7 \%$ of all cases), who were able to be back to their former work, had been transferred for medical rehabilitation within sixty postoperative days.

2. Two out of five postoperative cases of brain tumor developed recurrence during the period of medical rehabilitation. This would indicate the importance 